ARTíCUlo

\title{
Balance LOICZ de nitrógeno y fósforo para un área marina proglacial: Influencia de la variación pluviométrica en el flujo de nutrientes
}

LOICZ nitrogen and phosphorus budget in a proglacial marine area: Influence of pluviometric variation on nutrients flux

\begin{abstract}
Julio Salcedo-Castro ${ }^{\circledR 1,2,3^{*}}$, Américo Montiel $^{{ }^{4}}$ y Katherine Barría ${ }^{4}$
${ }^{1}$ The Sino-Australian Research Consortium for Coastal Management (SARCCM), University of New South Wales, Canberra, Australia ${ }^{2}$ Centro de Estudios Avanzados, Universidad de Playa Ancha, Traslaviña 450, Viña del Mar, Chile

${ }^{3}$ Laboratorio de Oceanografia, Clima y Procesos Ambientales (LOCPA), Universidad de Playa Ancha, Traslaviña 450, Viña del Mar, Chile

${ }^{4}$ Laboratorio de Ecología Funcional, Instituto de la Patagonia, Universidad de Magallanes, Avenida Bulnes 01890, Punta Arenas, Chile *Autor corresponsal: jusalca@gmail.com

Abstract.- The flow and balance of dissolved inorganic nitrogen and dissolved inorganic phosphorus in Seno Gallegos (Cordillera Darwin) was estimated for the first time. In addition, the response of these processes was evaluated under two rainfall conditions, one above and one below the local historical average. To estimate the flow and balance of nutrients, a LOICZ model of double layers was used. Primary data on nitrogen and phosphorus concentration were obtained from water samples, which were collected during fall, winter, and spring 2010 in Seno Gallegos. In the surface layer of the Seno Gallegos the balance of dissolved inorganic nitrogen showed a consumption and output of nutrients. While the vertical flow is predominantly from the deep layer to the surface one. The phosphorus balance of the Seno Gallegos was negative for the upper layer and positive in the deep layer. Under a condition of increased rainfall, nutrient balance and flow would be intensified, along with increased nitrogen fixation and net metabolism. While under a condition of lower rainfall, these processes would be diminished. Due to the direct relationship between variations of the glacial ice volume and its adjacent proglacial marine area, the latter are highly sensitive to climate change. Therefore, further studies on these areas are of great importance to understand the forces that govern these cold estuarine ecosystems.
\end{abstract}

Key words: Glaciomarine, nutrients, climate change, Patagonia

Resumen.- Se estimó por primera vez el flujo y balance de nitrógeno inorgánico disuelto y fósforo inorgánico disuelto en el Seno Gallegos (Cordillera Darwin). Además, se evaluó la respuesta de estos procesos bajo dos condiciones de pluviosidad, una por sobre y por debajo el promedio local histórico. Para estimar el flujo y balance de los nutrientes, se utilizó un modelo LOICZ de dos capas. Los datos primarios de concentración de nitrógeno y fósforo fueron obtenidos de muestras de agua, las cuales fueron recolectadas durante otoño, invierno y primavera del 2010 en el Seno Gallegos. En la capa superficial del Seno Gallegos el balance de nitrógeno inorgánico disuelto mostró un consumo y salida de nutrientes. Mientras que el flujo vertical es predominantemente desde la capa profunda hacia la superficial. El balance fósforo del Seno Gallegos fue negativo para la capa superior y positivo en la capa profunda. Bajo una condición de mayor pluviosidad, el balance y flujo de nutrientes se vería intensificado, junto con un incremento de la fijación de nitrógeno y metabolismo neto. Mientras que, bajo una condición de menor pluviosidad, estos procesos se verían disminuidos. Dada la relación directa entre las variaciones del volumen de hielo glaciar y las áreas marinas proglaciales, estas últimas son altamente sensibles al cambio climático, por lo que mayores estudios sobre estas áreas son de gran importancia para entender las fuerzas que gobiernan estos ecosistemas estuarinos fríos.

Palabras clave: Glaciomarino, nutrientes, cambio climático, Patagonia

\section{INTRODUCCIÓN}

La disponibilidad y flujo de nutrientes son fundamentales en los procesos biogeoquímicos, y la producción primaria; además, juegan un rol limitante en el ecosistema marino de los fiordos y canales australes (Pizarro et al. 2000, Ramírez 2005). Por lo tanto, conocer la dinámica y balance de nutrientes es imprescindible para entender el funcionamiento de los diferentes ecosistemas costeros.
El ecosistema de fiordos y canales chilenos es uno de los sistemas estuarinos más grandes del planeta (Silva \& Vargas 2014), abarca más de $1.000 \mathrm{~km}$ entre los $41^{\circ}$ y $55^{\circ}$ de latitud sur, con una superficie aproximada de $240.000 \mathrm{~km}^{2}$ y se caracteriza por una compleja dinámica biogeoquímica (Silva \& Palma 2008, González et al. 2010). En dicha región, existen numerosos fiordos con características proglaciales y glaciofluviales, los cuales 
están adyacentes a campos de hielo, glaciares de valle y de montaña (Kuylenstierna et al. 1996, Rivera et al. 2007, López et al. 2010, Slaymaker 2011). Sin embargo, la mayoría de los estudios oceanográficos se ha enfocado en fiordos de tipo glaciofluvial (con aporte de agua dulce por medio de ríos de origen glacial), como el fiordo Reloncaví (Guzmán \& Silva 2002, Valenzuela \& Avaria 2009, González et al. 2010, Calvete \& Sobarzo 2011, Montero et al. 2011, Pantoja et al. 2011, Castillo 2012, Schneider et al. 2014) y el fiordo Aysén (Silva et al. 1997, Guzmán \& Silva 2002, Prado-Fiedler \& Salcedo-Castro 2008). Mientras que, a partir del siglo XXI, los estudios oceanográficos en fiordos proglaciales han aumentado significativamente (Sievers et al. 2002, Valdenegro \& Silva 2003, Pizarro et al. 2005, Ramírez 2005, Silva 2006, Prado-Fiedler 2009, González et al. 2010, Quiroga et al. 2012, 2013; SalcedoCastro et al. 2015).

Desde un punto de vista oceanográfico, los fiordos proglaciares, además de ser determinante en la estratificación de temperatura y salinidad, el derretimiento del glaciar influye en características como la concentración de sólidos totales suspendidos, nutrientes, clorofila- $a$ y oxígeno disuelto (Faviano et al. 1999, Silva 2006, Torres et al. 2011, Vásquez et al. 2012, Salcedo-Castro et al. 2015). Los estudios sobre fiordos proglaciales se han enfocado principalmente en la descripción de características oceanográficas (Andrade 1991, Ramírez 2005, Torres et al. 2011, Vásquez et al. 2012, Haro et al. 2013). Sin embargo, las investigaciones sobre procesos asociados a la distribución y flujo de nutrientes son escasas. Por ejemplo, al sur del Estrecho de Magallanes solo se cuenta con estudios puntuales sobre las concentraciones de nutrientes (nitrito, nitrato, fosfato, y silicato) (Faranda et al. 1996, Iriarte et al. 2001, Valdenegro \& Silva 2003, Silva 2006). El Seno Ballena es otra área proglacial, donde la concentración de nitrato en la capa superior (hasta los 20 $\mathrm{m}$ de profundidad) varía entre $0,2-12 \mu \mathrm{M}$, la cual desciende hacia sectores cercanos al glaciar y aumenta a profundidades mayores de $10 \mathrm{~m}$ (Torres et al. 2011).

En este contexto, el balance y flujo de nutrientes en sistemas proglaciales no ha sido abordado hasta la fecha. El modelo desarrollado en el marco de Land Ocean Interactions in the Coastal Zone (LOICZ), es un modelo de balance de compartimientos que permite describir la dinámica de los procesos biogeoquímicos en zonas costeras semi-cerradas a distintas escalas geográficas (Gordon et al. 1996, Giordani et al. 2005, Ramesh et al. 2015). Su uso se ha enfocado principalmente en bahías y estuarios fluviales de ríos interurbanos que generalmente son utilizados como receptores de desechos sanitarios (Smith et al. 2000, Montalvo \& Loza 2006, Swaney \& Giordani 2011). En Chile, el fiordo Aysén es el único sistema marino, en el cual se ha aplicado el modelo LOICZ (Silva et al. 2000, Guzmán 2004). Posteriormente, Pantoja et al. (2010) aplicaron este modelo al sistema de fiordos entre $43^{\circ} \mathrm{S}$ y $46,5^{\circ} \mathrm{S}$, como una forma de determinar el balance y flujo de nutrientes entre la zona de fiordos y el océano.

Además de entender el balance actual de nutrientes y dado el rápido cambio que los ambientes proglaciales están experimentando durante las últimas décadas, es necesario evaluar su posible respuesta ante modificaciones del patrón de pluviosidad, asociadas al cambio climático. Se ha observado que los cambios en el régimen medio del patrón meteorológico inciden en el balance hídrico y biogeoquímica de los estuarios (Abreu et al. 2010, Cabral \& Fonseca 2019).

Por tanto, los objetivos del presente estudio fueron estimar por primera vez el flujo y balance de nitrógeno inorgánico disuelto (DIN) y fósforo inorgánico disuelto (DIP) en un área proglacial y evaluar las respuestas del balance de nitrógeno y fósforo disueltos bajo dos posibles condiciones de aumento y disminución de la pluviosidad con respecto al promedio local histórico, utilizando el modelo LOICZ. De esta manera, el presente estudio representa una primera aproximación a los complejos procesos de flujo de nutrientes en áreas marinas proglaciales subantárticas.

\section{MATERIALES Y MÉTODOS}

\section{ÁREA DE ESTUdio}

Se seleccionó como área de estudio el Seno Gallegos (54²3'37,4' 'S-6950'48,9'”), y uno de los numerosos fiordos que conforman el ambiente proglacial marino que conecta con el Estrecho de Magallanes, a través del Seno Almirantazgo, en Tierra del Fuego (Fig. 1). Este fiordo es un área marina proglacial ubicada adyacente al Campo de Hielo de Cordillera Darwin (Vásquez et al. 2012, Salcedo-Castro et al. 2015), la cual, con una altitud promedio de $612 \mathrm{~m} \mathrm{y}$ varios picos sobre los $2000 \mathrm{~m}$, impone un efecto orográfico importante en el régimen de vientos y precipitaciones (Fernández et al. 2011). En la cabeza de Seno Gallegos se ubica el glaciar Garibaldi, uno de los ocho glaciares provenientes del Campo de Hielo de la Cordillera Darwin (Homlund \& Fuenzalida 1995). Este glaciar es el principal tributario de agua dulce en el Seno Gallegos (Fig. 1).

Seno Gallegos mide alrededor de $9 \mathrm{~km}$ de largo, con un ancho promedio de $2,3 \mathrm{~km}$ y posee dos cuencas de profundidades máximas de 100 y $170 \mathrm{~m}$, respectivamente, separadas por un sill o un umbral con una profundidad de $50 \mathrm{~m}$ (Salcedo-Castro et al. 2015). La superficie del fiordo es de $21 \mathrm{~km}^{2}$ y tiene una profundidad promedio de $55 \mathrm{~m}$. Este fiordo presenta una capa superficial, caracterizada por temperaturas entre 4 y $9{ }^{\circ} \mathrm{C}$, una salinidad entre 23-30; y otra capa profunda, con temperaturas de entre 6 y $7^{\circ} \mathrm{C}$ y una salinidad de 29-30 (Vásquez 2011, Salcedo-Castro et al. 2015). 

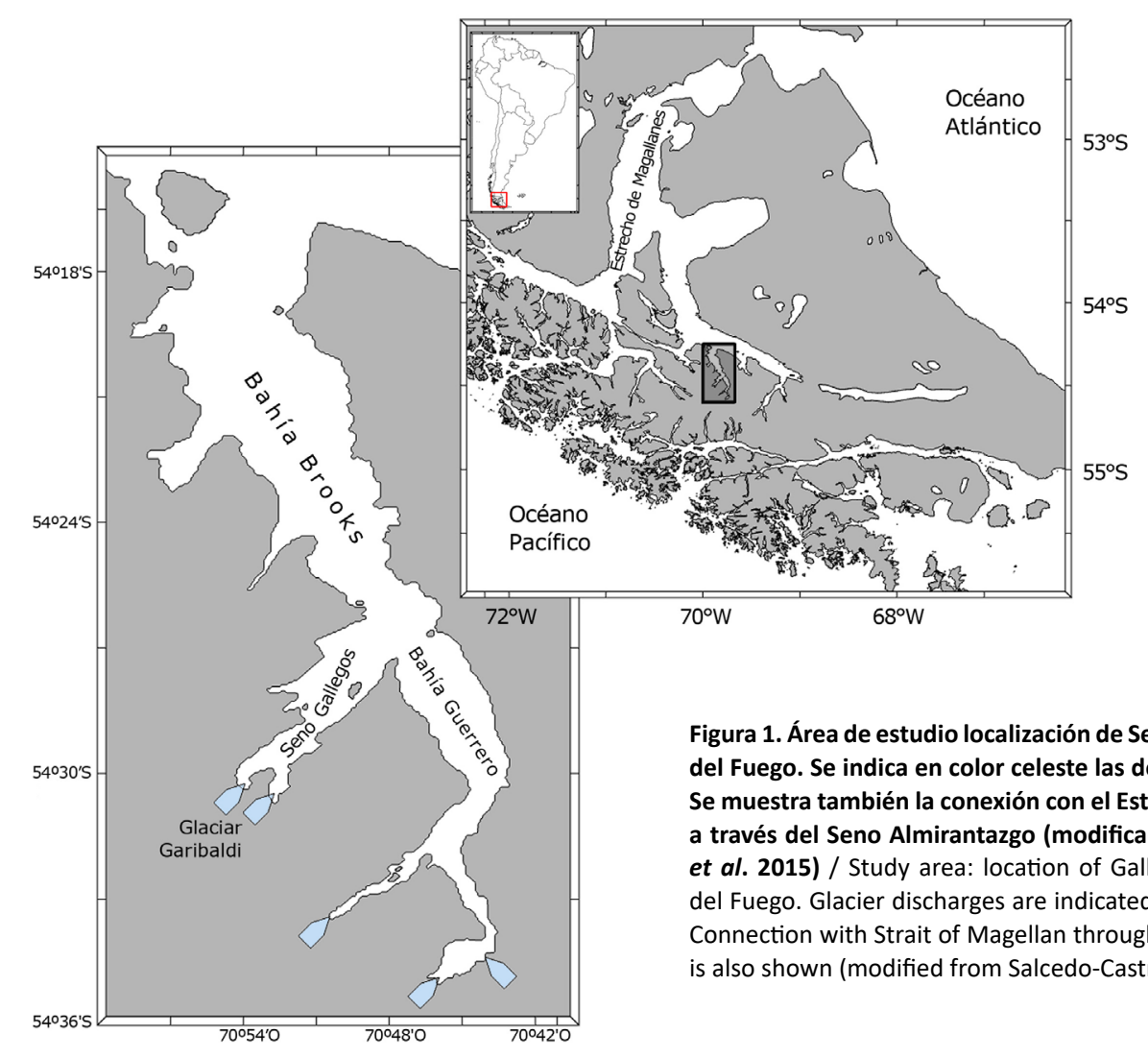

\begin{abstract}
Figura 1. Área de estudio localización de Seno Gallegos en Tierra del Fuego. Se indica en color celeste las descargas de glaciares. Se muestra también la conexión con el Estrecho de Magallanes, a través del Seno Almirantazgo (modificado de Salcedo-Castro et al. 2015) / Study area: location of Gallegos Sound at Tierra del Fuego. Glacier discharges are indicated with light blue color. Connection with Strait of Magellan through Almirantazgo Sound is also shown (modified from Salcedo-Castro et al. 2015)
\end{abstract}

\section{CONFIGURACIÓN MODELO LOICZ}

Para la estimación del balance y flujo de nutrientes, se utilizó el modelo Land Ocean Interactions in the Coastal Zone (LOICZ). Este modelo ha demostrado ser adecuado para el estudio de ambientes acuáticos semi-cerrados, mediante la compartimentalización del sistema. La ventaja de estos modelos de caja es que permiten utilizar información dispersa de diversas fuentes y entregar estimaciones de balance y flujo de nutrientes, en comparación a los modelos determinísticos que normalmente requieren de una mayor cantidad de información, tiempo y altos recursos computacionales (Gordon et al. 1996, Guzmán 2004, Giordani et al. 2005). Como consecuencia, cuando se requiere tener una visión rápida de los cambios que puedan ocurrir en un sistema marino y no se dispone de información detallada (series de tiempo de correntómetros, de caudales, evaporación, precipitación, vientos, mareas, etc.), el uso de LOICZ ha sido altamente recomendado. La aproximación con modelos de balance de compartimientos puede entregar información de primera instancia para interpretar el efecto que pueden producir cambios inducidos por forzantes y procesos químicos, biológicos y geológicos (Smith et al. 2000, Montalvo \& Loza 2006). Seno Gallegos es un pequeño fiordo estratificado, con un principal aporte de agua dulce representado por el derretimiento glaciar, por lo que este modelo simplificado permite hacer estimaciones aceptables de los procesos biogeoquímicos que ocurren en el fiordo. A pesar que es difícil estimar la incertidumbre en las estimaciones con la información disponible, estudios previos han demostrado que LOCIZ es un modelo lo suficientemente robusto en sus supuestos y estimaciones (Gordon et al. 1996, Giordani et al. 2005, Ramesh et al. 2015).

Para la determinación de los flujos y balance de nutrientes se utilizó LOICZ-toolbox, una planilla Excel que integra las diferentes ecuaciones para la determinación de los flujos y balances, a través de macros (Swaney \& Hong 2009a, b). La modelación consistió en estimar el balance y flujo de nutrientes entre Seno Gallegos y el Estrecho de Magallanes. La configuración vertical utilizada fue de dos capas. El límite inferior de la capa superficial se determinó en base a la posición de la haloclina y la frecuencia de Brunt-Väisälä, a partir de perfiles de CTD, utilizando el programa Ocean Data View (ODV) versión 4 (Schlitzer 2016). Este límite se presentó a $5 \mathrm{~m}$, similar a las descripciones previas (Vásquez 2011, Salcedo-Castro et al. 2015). El área y volumen del sistema fueron estimadas con el programa Surfer ${ }^{\circledR}$, versión 10 (Golden Software, LLC), a partir de la digitalización de la carta náutica SHOA 12.500 y la batimetría del fiordo (Salcedo-Castro et al. 2015). 
La configuración del sistema para el balance y flujo de DIN y DIP se muestra en la Figura 2. Dentro de los términos asociados al balance de nutrientes dentro de cada compartimento y los flujos verticales y horizontales, N FijDenit corresponde a la diferencia entre el $\triangle D I N$ esperado y el estimado, donde el $\Delta \mathrm{DIN}$ esperado es $\Delta \mathrm{DIP}$ multiplicado por 16 , de acuerdo a la razón de Redfield $(\mathrm{N}: \mathrm{P}=16)$. Este término representa la transferencia entre los compuestos de nitrógeno fijado (nitrógeno orgánico e inorgánico disuelto y nitrógeno particulado) y el nitrógeno gaseoso (Smith et al. 2000). El metabolismo neto del ecosistema, correspondiente a la diferencia producción-respiración ( $p$ r), es estimado también a partir de la relación de Redfield $(\mathrm{C}: \mathrm{P}=106)$, como -106 x $\Delta \mathrm{DIP}$ (Gordon et al. 1996).

Para estimar el balance de nutrientes, el nitrógeno inorgánico disuelto (DIN) correspondió a la suma nitrato+nitrito, mientras que el fósforo inorgánico disuelto (DIP) correspondió a la concentración de ortofosfato. Estos valores fueron obtenidos de los resultados correspondientes a las campañas realizadas en Seno Gallegos durante otoño, invierno y primavera del 2010 (Barría 2016). Los valores de nutrientes en el Estrecho de Magallanes se obtuvieron de Faranda et al. (1996) y de los cruceros oceanográficos Cimar 3 Fiordos y Cimar 16 Fiordos (CENDHOC 2010). En ambos casos, se calculó el promedio de los valores de nutrientes para tener un valor representativo para cada capa.
Los aportes y flujos correspondientes a otros aportes de agua dulce $\left(\mathrm{V}_{\mathrm{r}}\right)$, flujo de agua subterráneas $(\mathrm{Vg})$, flujo de nitrógeno y fósforo de aguas servidas, concentración de nitrógeno (DIN) de la precipitación (DINp), se asumieron despreciables (Valores $=0$ ), según las recomendaciones de otros autores (Gordon et al. 1996, Silva et al. 2000, Guzmán 2004 y Giordani et al. 2005).

\section{ESTIMACIÓN DE APORTE de AgUa dULCE POR DERRETIMIENTO GLACIAL}

La principal característica de un fiordo glacial es que el mayor volumen de agua dulce proviene del derretimiento del glaciar. En este caso, el principal aporte de agua dulce en el Seno Gallegos es el agua de derretimiento del glaciar Garibaldi. Para tener una estimación del aporte de agua dulce generado por el derretimiento glacial, se aplicó el método de degree-day factor (Hock 2003), usando los coeficientes de derretimiento de nieve $\left(0,0035 \mathrm{~m}\right.$ w.e. ${ }^{\circ} \mathrm{C}^{-1}$ $\left.\mathrm{d}^{-1}\right)$ e hielo $\left(0,0065 \mathrm{~m}\right.$ w.e. $\left.{ }^{\circ} \mathrm{C}^{-1} \mathrm{~d}^{-1}\right)$ propuestos por De Angelis (2014), junto con el área del glaciar $\left(53,15 \mathrm{~km}^{2}\right)$ (DGA 2016) ${ }^{1}$. Para realizar los cálculos de derretimiento, se calculó el valor promedio a partir de los valores mensuales de temperatura del aire durante los meses en que se realizaron las campañas oceanográficas. Debido a que no existen registros meteorológicos en Seno Gallegos, se eligieron tres estaciones meteorológicas representativas en la zona de estudio. A partir de estas estaciones, se estimó una precipitación promedio anual de $60 \mathrm{~mm}$, la cual es consistente con la descripción de Fernández et al. (2011).

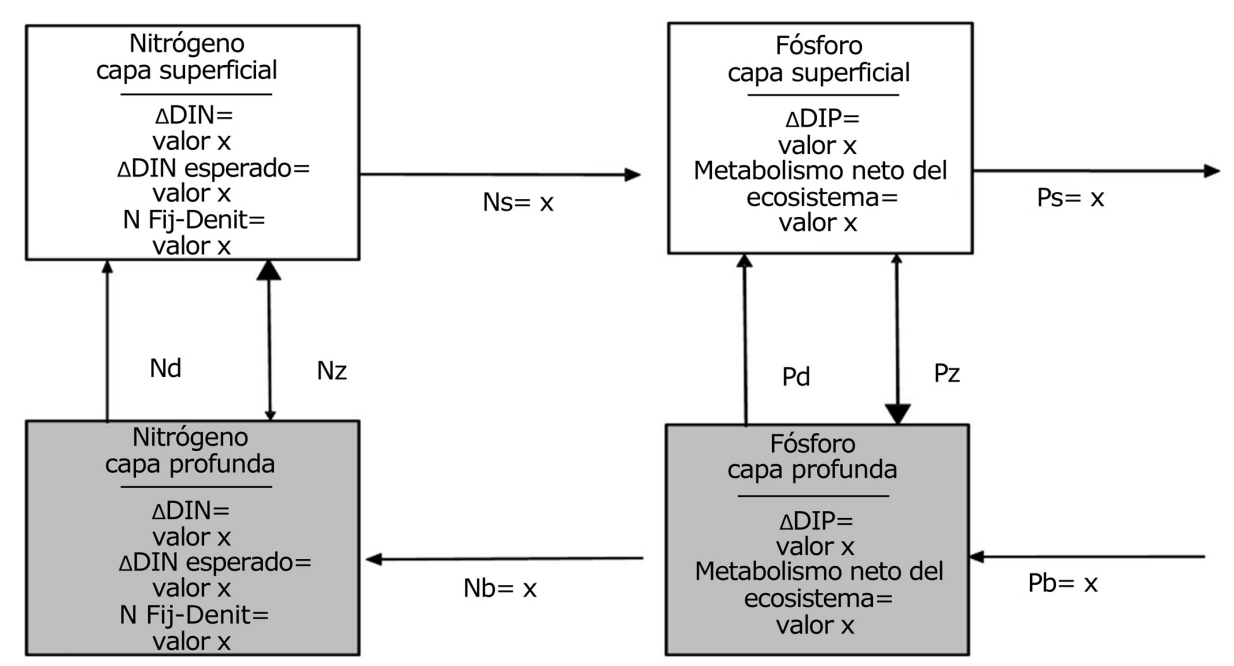

Figura 2. Ejemplo de diagramas de compartimientos creados con LOICZ toolbox para un sistema de dos capas, para nitrógeno inorgánico disuelto (izquierda) y fósforo inorgánico disuelto (derecha) / Example of box diagrams created with LOICZ toolbox for a two-layer system, for dissolved inorganic nitrogen (left) and dissolved inorganic phosphorus (right) 


\section{MODELAMIENTO DE ESCENARIOS HÚMEDO Y SECO}

Para evaluar la respuesta de Seno Gallegos ante diferentes condiciones de precipitación, se simuló el flujo de nutrientes bajo dos posibles escenarios: un clima húmedo (precipitación anual media: $86 \mathrm{~mm}$ ) y un clima seco (precipitación anual media: $17 \mathrm{~mm}$ ). En ambos casos, se mantuvo el valor promedio de temperatura actual, ya que exhibe menor variabilidad $\left(2-4{ }^{\circ} \mathrm{C}\right)$ en Campos de Hielo de Cordillera Darwin, en comparación a los cambios interanuales e interdecadales en el régimen precipitación (Fernández et al. 2011).

Para estimar los posibles escenarios de pluviosidad en la zona de estudio, se utilizaron datos promedio desde el 2011 al 2015 de dos zonas distintas: Río Azopardo y Monte Aymond, representativas de un clima más húmedo (mayor pluviosidad) y más seco (menor pluviosidad), respectivamente. Los datos de precipitación fueron obtenidos de la base de datos de la Dirección General de Aguas (DGA) ${ }^{1}$. La ubicación de estas estaciones se muestra en la Figura 3.

\section{RESUlTados}

\section{BALANCE Y FLUJO DE NUTRIENTES BAJO CONDICIONES ACTUALES}

El balance de nitrógeno inorgánico en la capa superior fue de $-33045 \mathrm{~mol} \mathrm{~d}^{-1}$ ( $\triangle \mathrm{DIN}$ esperado de $-1232597 \mathrm{~mol} \mathrm{~d}^{-1}$ ), mientras que la diferencia N Fij-Denit fue de 1199552 mol d $\mathrm{d}^{-1} \mathrm{y}$ un flujo horizontal de salida (Ns) de -76795 $\mathrm{d}^{-1}$. En la capa profunda, el $\triangle \mathrm{DIN}$ fue de $23347 \mathrm{~mol} \mathrm{~d}^{-1}$ ( $\Delta$ DIN esperado de $1203505 \mathrm{~mol} \mathrm{~d}^{-1}$ ), el N Fij-Denit fue de $-1180157 \mathrm{~mol} \mathrm{~d}^{-1}$, mientras que el flujo horizontal $(\mathrm{Nb}) \mathrm{de}$ entrada fue de $86492 \mathrm{~mol} \mathrm{~d}^{-1}$. Por otra parte, el flujo vertical entre la capa superficial y profunda en Seno Gallegos (Nd) fue de $97233 \mathrm{~mol} \mathrm{~d}^{-1}$, mientras que la diferencia entre los flujos de ambos sistemas (DIN sistema prof $-\mathrm{DIN} \mathrm{sistema} \mathrm{superf})$ fue de $12607 \mathrm{~mol} \mathrm{~d}^{-1}$ (Fig. 4).

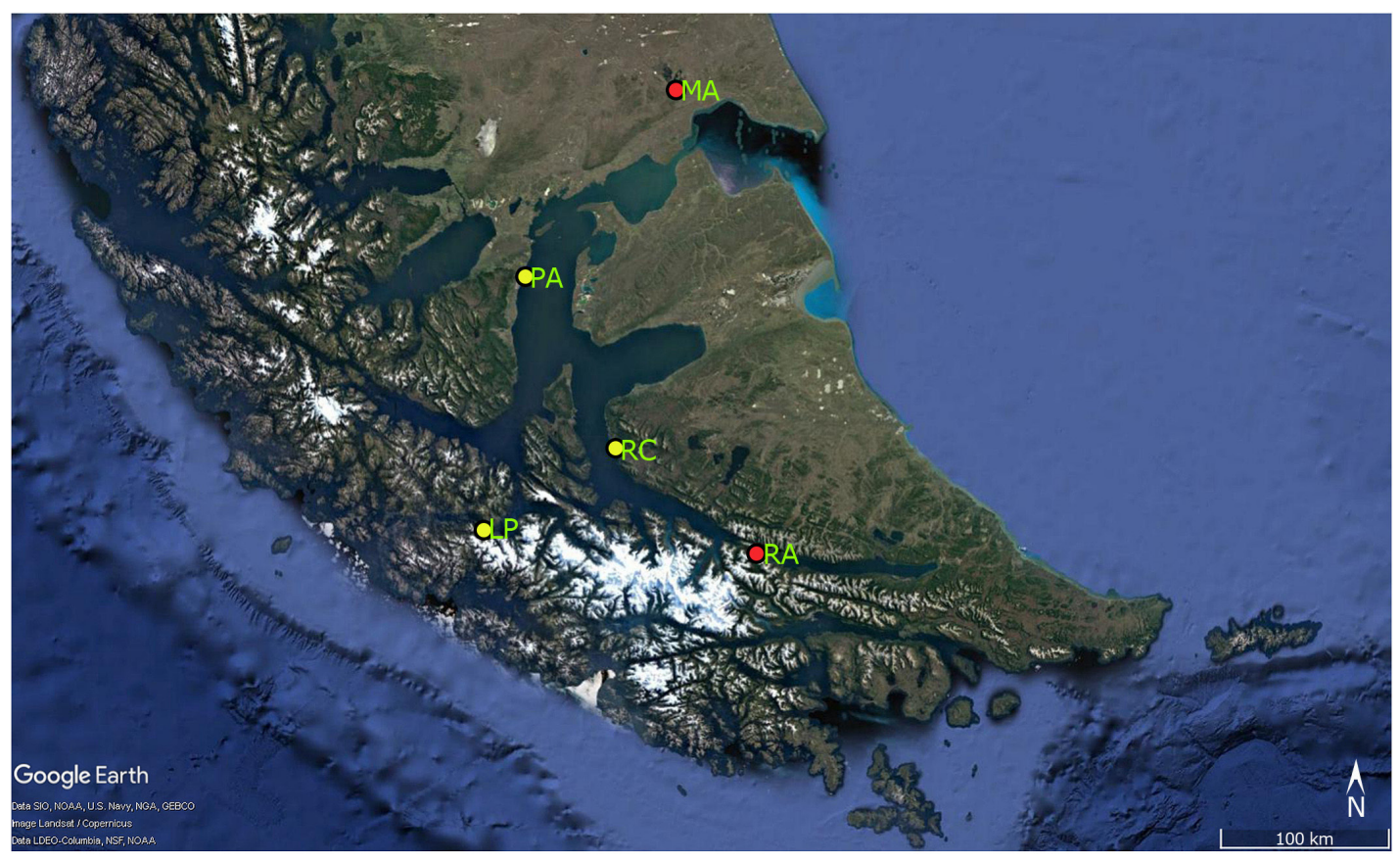

Figura 3. Ubicación de las estaciones meteorológicas en Seno Gallegos ( $\left.54^{\circ} 23^{\prime} 37,4^{\prime \prime} \mathrm{S}-69^{\circ} 50^{\prime} 48,9^{\prime \prime O}\right)$ (Cordillera Darwin, Chile). En círculos amarillos se identifican las estaciones para la estimación de temperatura y precipitación promedio actual [Punta Arenas (PA), Río Cóndor (RC) and Laguna Parrillar (LP)]. En círculos rojos, se muestran las estaciones que representan condiciones más húmedas (Río Azopardo, RA) y más secas (Monte Aymond, MA) que las actuales (para más información sobre las características locales de precipitación, ver fig. 3 en Fernández et al. 2011) / Location of weather stations used in this study from Seno Gallegos ( $54^{\circ} 23^{\prime} 37.4^{\prime \prime S}-69^{\circ} 50^{\prime} 48.9^{\prime \prime} \mathrm{W}$ ) (Cordillera Darwin, Chile). Stations to estimate annual mean temperature and precipitation are shown in yellow circles [Punta Arenas (PA), Río Cóndor (RC) and Laguna Parrillar (LP)]. Stations that represent more humid (Río Azopardo RA) and drier (Monte Aymond MA) conditions are shown in red circles (for more information about local precipitation characteristics, see fig. 3 in Fernández et al. 2011) 

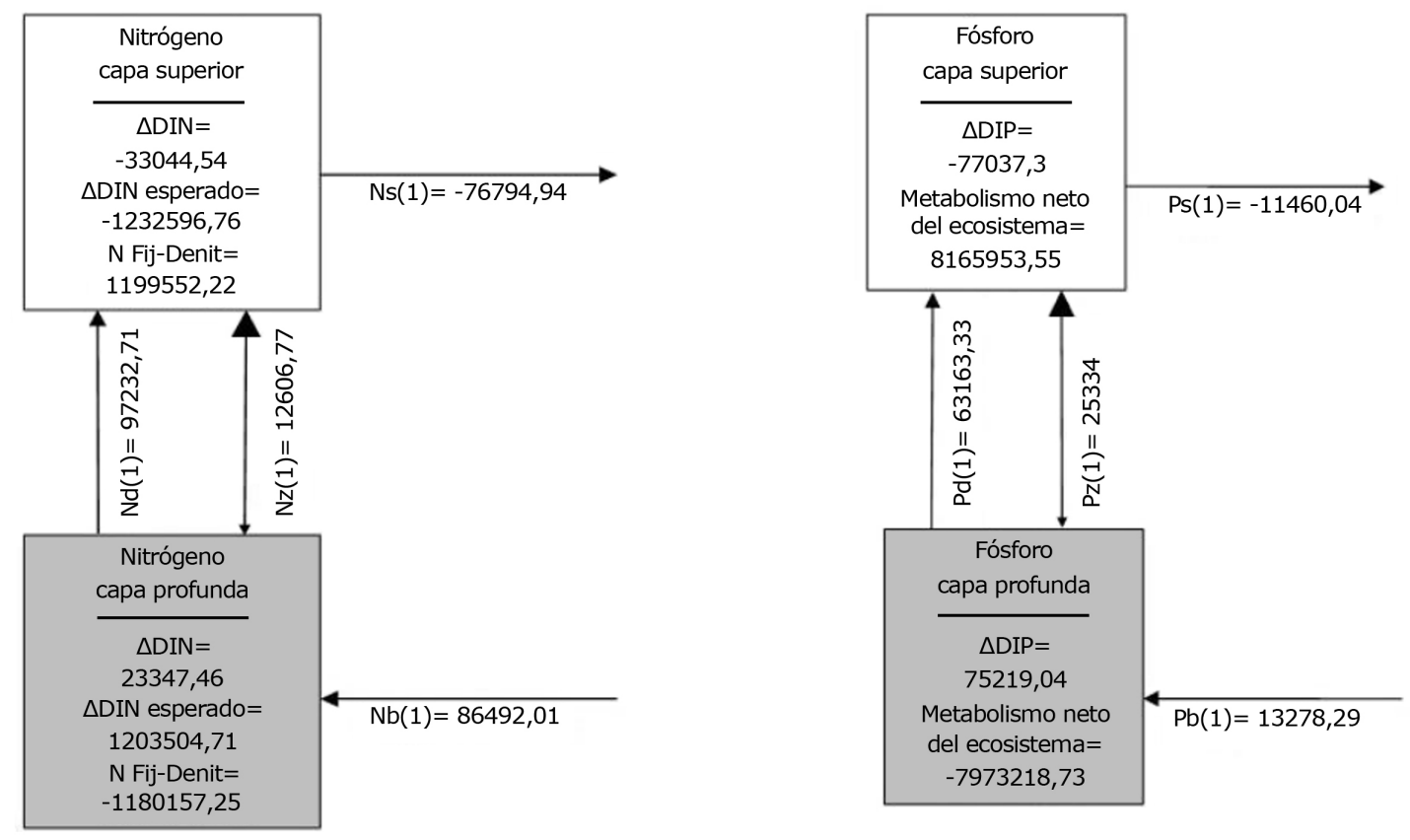

Figura 4. Balance de DIN (izquierda) y DIP (derecha) en Seno Gallegos. También se muestran los flujos entre las capas superficial y profunda / DIN (left) and DIP (right) budget in Gallegos Sound. Fluxes between the surface and deep layer are also shown

El balance fósforo inorgánico disuelto $(\triangle \mathrm{DIP})$ en la capa superior se comportó de manera negativa $(-77037 \mathrm{~mol}$ $\mathrm{d}^{-1}$ ), con un metabolismo neto de $8165954 \mathrm{~mol} \mathrm{C} \mathrm{d}^{-1} \mathrm{y}$ un flujo horizontal de $-11460 \mathrm{~mol} \mathrm{~d}^{-1}$. En la capa profunda, el $\triangle$ DIP fue positivo (75219 $\mathrm{mol} \mathrm{d}^{-1}$ ), con un metabolismo neto de $-7973219 \mathrm{~mol} \mathrm{C} \mathrm{d}^{-1}$ y un flujo horizontal de 13278 $\mathrm{mol} \mathrm{d}^{-1}$. En tanto, el flujo vertical entre la capa superficial y profunda $(\mathrm{Pd})$ fue de $63163 \mathrm{~mol} \mathrm{~d}^{-1}$, mientras que la diferencia de flujos verticales (DIP ${ }_{\text {sistema prof }}$-DIP ${ }_{\text {sistema superf }}$ ) fue de $25334 \mathrm{~mol} \mathrm{~d}^{-1}$ (Fig. 4).

\section{BALANCE Y FLUJO DE NUTRIENTES BAJOS ESCENARIOS SECO Y HÚMEDO SIMULADOS}

\section{NitRóGENO INORGÁNICO DISUELTO}

Bajo una condición de clima húmedo, con mayor pluviosidad, el balance $\triangle \mathrm{DIN}$ en la capa superior fue de -33754 $\mathrm{mol} \mathrm{d}^{-1}$, con un $\triangle \mathrm{DIN}$ esperado de $-1259023 \mathrm{~mol}$ $\mathrm{d}^{-1}$, una diferencia N Fij-Denit de $125270 \mathrm{~mol} \mathrm{~d}^{-1}$ y un flujo horizontal de salida (Ns) de -78441. El balance $\triangle \mathrm{DIN}$ en la capa de fondo fue de $23848 \mathrm{~mol} \mathrm{~d}^{-1}$, con un $\triangle \mathrm{DIN}$ esperado de $1229307 \mathrm{~mol} \mathrm{~d}^{-1}$, un desbalance N Fij-Denit de -12054059 mol d$^{-1}$ y un flujo horizontal $\mathrm{Nb}$ de entrada que fue positivo ( $\left.88346 \mathrm{~mol} \mathrm{~d}^{-1}\right)$. En la dimensión vertical del sistema Seno Gallegos, el flujo vertical entre la capa superficial y profunda fue de $99317 \mathrm{~mol} \mathrm{~d}^{-1}$, mientras que la diferencia de flujos (Nz) fue $12877 \mathrm{~mol} \mathrm{~d}^{-1}$ (Fig. 5).
En un escenario de clima seco, de menor pluviosidad, la diferencia $\triangle \mathrm{DIN}$ en la capa superior fue de -31980 mol d $\mathrm{d}^{-1}$ ( $\Delta$ DIN esperado: $-1192898 \mathrm{~mol} \mathrm{~d}^{-1}$ ), con un flujo horizontal Ns negativo (-74322 $\left.\mathrm{mol} \mathrm{d}^{-1}\right)$ mientras que el proceso N Fij-Denit fue de $1160918 \mathrm{~mol} \mathrm{~d}^{-1}$. En la capa de fondo, la diferencia $\triangle \mathrm{DIN}$ fue $22596 \mathrm{~mol} \mathrm{~d}^{-1}$, con un $\triangle \mathrm{DIN}$ esperado de $1164743 \mathrm{~mol} \mathrm{~d}^{-1}$, una diferencia N Fij-Denit de $-1142148 \mathrm{~mol} \mathrm{~d}^{-1}$ y un flujo horizontal $\mathrm{Nb}$ de entrada positivo $\left(83706 \mathrm{~mol} \mathrm{~d}^{-1}\right)$. El flujo vertical entre la capa superficial y profunda fue de $94101 \mathrm{~mol} \mathrm{~d}-1$, mientras que $\mathrm{Nz}$ fue de $12201 \mathrm{~mol} \mathrm{~d}^{-1}$ (Fig. 5).

\section{FóSFORO INORGÁNICO DISUELTO}

Bajo un escenario de clima húmedo, el balance $\triangle \mathrm{DIP}$ en la capa superior se comportó de manera negativa (-786889 $\mathrm{mol} \mathrm{d}^{-1}$ ), exhibiendo un metabolismo neto de $8341024 \mathrm{~mol}$ $\mathrm{C} \mathrm{d}^{-1} \mathrm{y}$ un flujo horizontal de salida de $-11706 \mathrm{~mol} \mathrm{~d}^{-1}$. En la capa profunda, el balance $\triangle \mathrm{DIP}$ fue de $76831 \mathrm{~mol} \mathrm{~d}^{-1}$, con un metabolismo neto de $-8144157 \mathrm{~mol} \mathrm{C} \mathrm{d}^{-1} \mathrm{y}$ un flujo horizontal de entrada (Ps) de $13563 \mathrm{~mol} \mathrm{~d}^{-1}$. El flujo vertical (Pd) entre la capa superficial y profunda de Seno Gallegos fue de $64518 \mathrm{~mol} \mathrm{C} \mathrm{d}^{-1}$, mientras que la diferencia de flujos (Pz) fue de $25877 \mathrm{~mol} \mathrm{C} \mathrm{d}^{-1}$ (Fig. 6). 

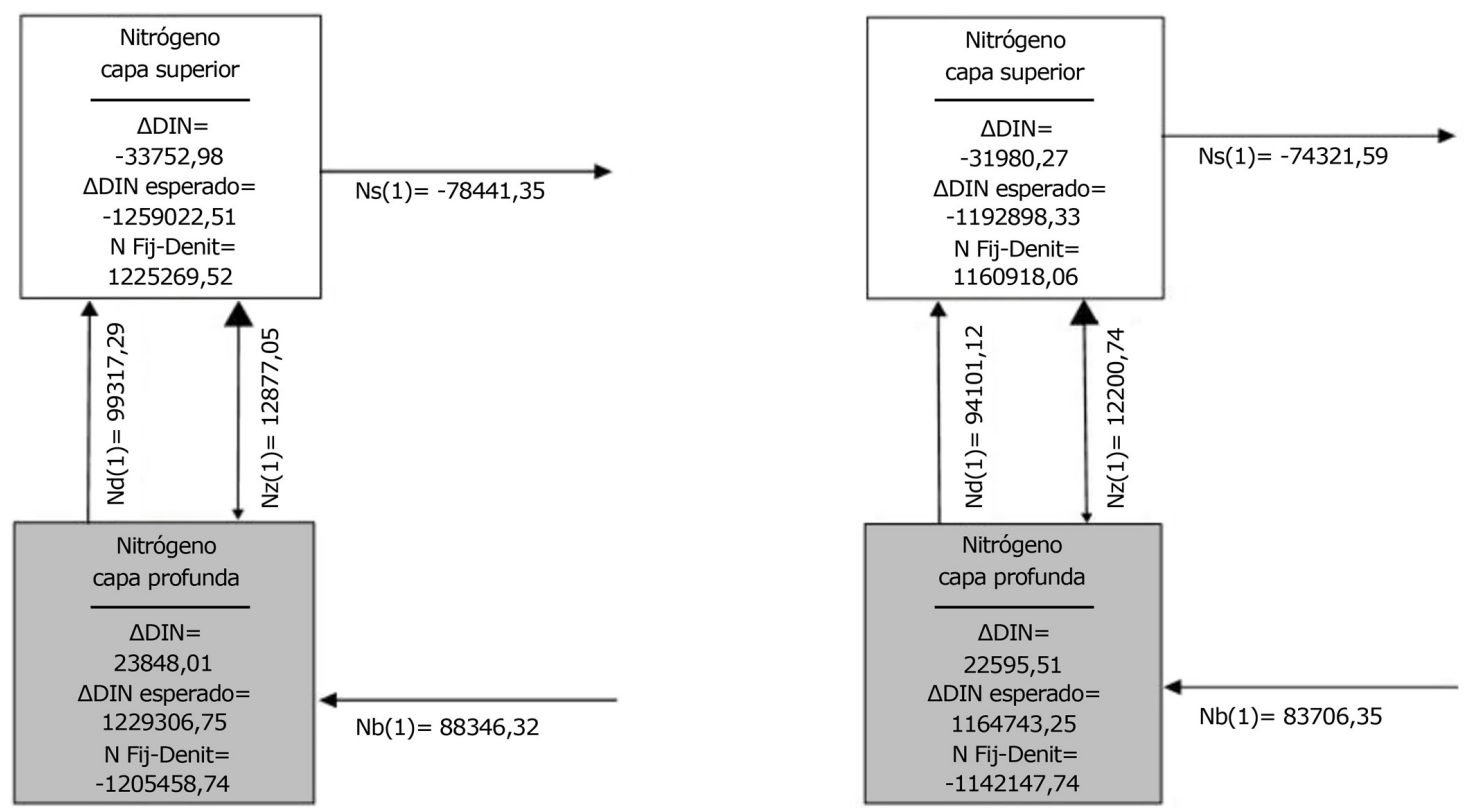

Figura 5. Balance de DIN en Seno Gallegos bajo un escenario de clima húmedo (izquierda) y seco (derecha) / DIN budget in Gallegos Sound under a scenario of humid (left) and dry (right) climate
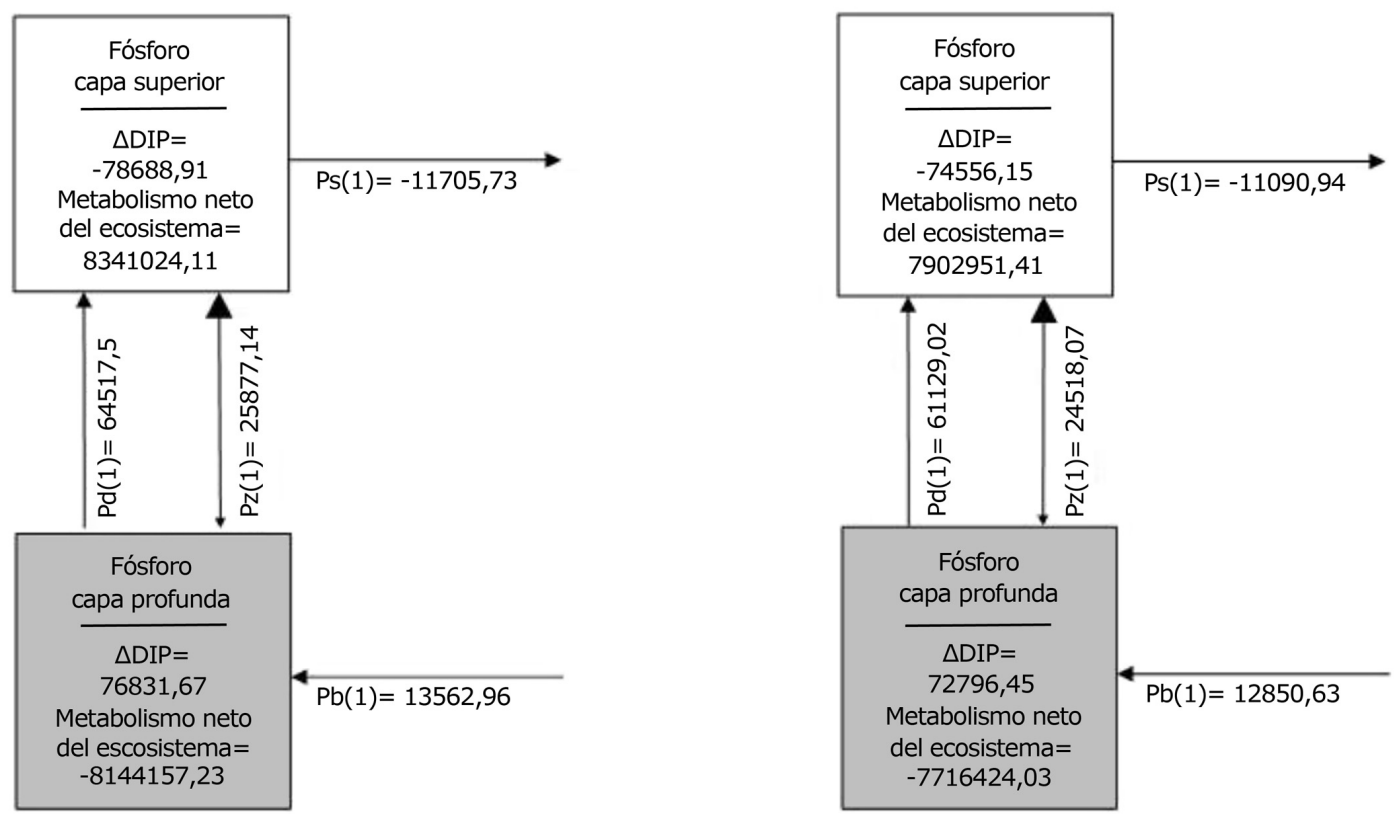

Figura 6. Balance de DIP en Seno Gallegos bajo un escenario de clima húmedo (izquierda) y seco (derecha) / DIP budget in Gallegos Sound under a scenario of humid (left) and dry (right) climate 
En condiciones de clima seco, el balance $\triangle \mathrm{DIP}$ en la capa superior fue negativo $\left(-74556 \mathrm{~mol} \mathrm{~d}^{-1}\right)$, con un flujo horizontal de salida de $-11091 \mathrm{~mol} \mathrm{~d}^{-1} \mathrm{y}$ un metabolismo neto de $790295 \mathrm{~mol} \mathrm{C} \mathrm{d}^{-1}$. El balance $\Delta$ DIP en la capa de fondo fue positivo (72796 $\left.\mathrm{mol} \mathrm{d}^{-1}\right)$, con un metabolismo neto de $-7716424 \mathrm{~mol} \mathrm{C} \mathrm{d}^{-1} \mathrm{y}$ un flujo horizontal positivo (12851 $\left.\mathrm{mol} \mathrm{d}^{-1}\right)$. El flujo vertical de DIP entre la capa superficial y profunda fue de $61129 \mathrm{~mol} \mathrm{C} \mathrm{d}^{-1}$, con un $\mathrm{Pz}$ de $24518 \mathrm{~mol} \mathrm{C} \mathrm{d}^{-1}$ (Fig. 6).

\section{Discusión}

\section{ANÁLISIS DE LOICZ EN DOS CAPAS}

Al realizar el análisis LOICZ de doble capa para al Seno Gallegos, se determinó que en la capa superficial el balance de nitrógeno inorgánico disuelto mostró un consumo y salida de nutrientes, mientras que el flujo vertical es predominante desde la capa profunda hacia la superficial; esto implica una mayor entrada de aguas oceánicas, más ricas en nutrientes de origen marino, en caso de este estudio provenientes desde el Estrecho de Magallanes. En términos cualitativos, el balance de nitrógeno disuelto modelado para el fiordo Aysén mostró un comportamiento similar al observado en Seno Gallegos (Silva et al. 1997, Guzmán 2004). Por otra parte, Pantoja et al. (2010) describieron el mismo patrón con el balance calculado para todos los fiordos entre $43^{\circ} \mathrm{S}$ y $46,5^{\circ} \mathrm{S}$. Similar al balance del nitrógeno, el balance $\triangle \mathrm{DIP}$ del Seno Gallegos fue negativo para la capa superior y positivo en la capa profunda, lo que confirma la importancia de aguas oceánicas en el aporte de nutrientes. Este proceso puede ser especialmente importante en fiordos glaciales, donde se observa pérdida de fósforo particulado asociado a la alta tasa de sedimentación y floculación en estos sistemas (Salcedo-Castro et al. 2013).

La relación $\mathrm{N}_{\text {fij }}-\mathrm{D}_{\text {enit }}$ fue positiva en la capa superficial, implicando fijación de nitrógeno que podría estar siendo realizada por bacterias; mientras que la capa profunda exhibió una relación $\mathrm{N}_{\text {fij }}-\mathrm{D}_{\text {enit }}$ negativa, mostrando el predominio de procesos de desnitrificación bajo la picnoclina. Guzmán (2004) describe el mismo resultado para el fiordo Aysén. Sin embargo, Pantoja et al. (2010) señalan que el sistema es altamente variable y es probable que estos procesos no sean estáticos y varíen anualmente.

El metabolismo neto (p-r) modelado para el Seno Gallegos presentó un estado trófico autótrofo (valor positivo) en la capa superior y heterótrofo (valor negativo) en la capa profunda. Esto corrobora el predominio de consumo de fósforo en la capa superficial y regeneración de fósforo en la capa profunda. Si se asume que la razón de Redfield es conservativa en los fiordos chilenos, el Seno Gallegos exhibe una condición autótrofa. En este sentido, varios estudios en fiordos de la Patagonia norte han mostrado la misma tendencia (Silva et al. 1997, Guzmán 2004, Pantoja et al. 2010).
La relación entre el flujo de nutrientes y el metabolismo neto muestran la conexión entre la biogeoquímica del sistema y forzantes externos, como la pluviosidad. En este sentido, la respuesta del fiordo glacial a forzantes atmosféricos ya ha sido demostrada por Salcedo-Castro et al. (2015), donde se indica la sincronía entre temperatura del aire y las características hidrográficas y productividad primaria (clorofila $a$ ) en Seno Gallegos. Esta influencia del cambio en las condiciones meteo-oceanográficas ha sido descrita recientemente, mediante la aplicación del modelo LOICZ en estuarios de Brasil, donde se observó una correlación entre la mayor lluvia y viento causados por el paso de tormentas y el grado de mezcla, flujo de nutrientes y estado trófico del sistema (Abreu et al. 2010, Cabral \& Fonseca 2019).

Es interesante señalar que el balance de nutrientes con baja relación DIN/DIP parece ser un patrón común en Seno Gallegos y otros fiordos patagónicos más septentrionales de la región. Esto se debe a que las aguas continentales que llegan a estos sistemas tienen una razón N:P menor a la razón de Redfield. Sin embargo, también se ha observado limitación por fósforo en la cabeza de algunos fiordos (Prado-Fiedler \& Salcedo-Castro 2008). Otra posible razón es que no se estén considerando otras formas de nitrógeno inorgánico, como el amonio (Pantoja et al. 2010), el cual se puede encontrar en concentraciones iguales o mayores que la suma nitrato+nitrito (Prado-Fiedler \& SalcedoCastro 2008).

\section{Pronóstico del análisis de LOICZ bajo dos CONDICIONES SIMULADAS DE PLUVIOSIDAD}

En este estudio se mostró que, bajo un clima eventualmente más lluvioso, el balance y flujo de nutrientes presentaría una mayor magnitud en ambas capas, con una intensificación de la fijación de nitrógeno en superficie y de las condiciones desnitrificantes en la capa de fondo, aproximándose a lo observado en fiordos fluviales de la nordpatagonia (Silva et al. 2000, Guzmán 2004, Pantoja et al. 2010). Una situación similar se observa para el caso del metabolismo neto (p-r), donde la condición de autotrofía se vería incrementada en superficie, mientras el estado de heterotrofía se acentuaría en la capa de fondo (Tabla 1) (Silva et al. 1997, Guzmán 2004). Sin embargo, este escenario dependerá de cuán conservativo sea el sistema, ya que su dinámica no siempre es tan estable respecto a la proporción de los nutrientes (Pantoja et al. 2010). Bajo un clima de menor precipitación, por otra parte, el balance y flujo de nutrientes presentaría una menor magnitud en ambas capas, con una disminución de la fijación de nitrógeno en superficie y desnitrificación en la capa de fondo, reflejando un contraste similar a las diferencias estacionales observadas en el fiordo Aysén (Sievers \& Prado 1994). 
Tabla 1. Comparación del balance y flujo de nutrientes en el Seno Gallegos, respecto a escenarios de clima húmedo y clima seco / Comparison of nutrients budget and fluxes in Gallegos Sound, with respect to more humid and dryer climate scenarios

\begin{tabular}{clrrr}
\hline \multirow{2}{*}{ Capa } & \multicolumn{1}{c}{ Nutrientes } & $\begin{array}{c}\text { Situación } \\
\text { actual }\end{array}$ & $\begin{array}{r}\text { Clima } \\
\text { húmedo }\end{array}$ & \multicolumn{1}{c}{$\begin{array}{c}\text { Clima } \\
\text { seco }\end{array}$} \\
\hline Superior & $\Delta \mathrm{DIN}\left(\mathrm{mol} \mathrm{d}^{-1}\right)$ & -33044 & -33753 & -31980 \\
& $\Delta \mathrm{DIP}\left(\mathrm{mol} \mathrm{d}^{-1}\right)$ & -77037 & -78689 & -74556 \\
& N Fij-Denit $\left(\mathrm{mol} \mathrm{d}^{-1}\right)$ & 1199552 & 1225269 & 1160918 \\
& $(\mathrm{p}-\mathrm{r})\left(\mathrm{mol} \mathrm{C} \mathrm{d}^{-1}\right)$ & 8165953 & 8341024 & 7902951 \\
Profunda & $\Delta \mathrm{DIN}\left(\mathrm{mol} \mathrm{d}^{-1}\right)$ & 23447 & 23848 & 22595 \\
& $\Delta \mathrm{DIP}\left(\mathrm{mol} \mathrm{d}^{-1}\right)$ & 75219 & 76832 & 72796 \\
& $\left.\mathrm{~N} \mathrm{Fij-Denit} \mathrm{(mol} \mathrm{d}{ }^{-1}\right)$ & -1180157 & -1205459 & -1142148 \\
& (p-r) $\left(\mathrm{mol} \mathrm{C} \mathrm{d}^{-1}\right)$ & -7973219 & -8144157 & -7716424 \\
\hline
\end{tabular}

Si bien bajo diferentes condiciones de pluviosidad se observan diferencias en el balance y flujo de DIN y DIP, estos valores no afectan la dinámica del Seno Gallegos en sí; es decir, tanto en un clima húmedo como en un clima seco, en el Seno Gallegos se presentaría la misma dinámica en relación con los flujos negativos en la capa superior y positivo en la capa inferior, lo que hace inferir que las precipitaciones no serían un factor determinante en la dinámica de nutrientes del Seno Gallegos.

El modelamiento de los flujos de nutrientes permite hacer inferencias respecto a los efectos de diferentes condiciones climáticas en la biogeoquímica de los sistemas glaciomarinos de Tierra del Fuego a futuro, así como explicar su influencia en climas pasados. Por ejemplo, esta alternancia de condiciones húmeda/seca ya ha sido observada en registros paleoclimáticos, a través de cambios vegetacionales por Mansilla et al. (2018), quienes señalan que los cambios en un gradiente longitudinal están asociados a cambios en la ocurrencia y cambios latitudinales en la banda de vientos de la deriva del oeste. Borromei et al. (2018) confirman esta aseveración, indicando que los cambios han sido de corta y larga escala temporal. En este sentido, durante las últimas décadas, Garreaud et al. (2013) describe un aumento moderado de las precipitaciones en esta región, en contraste a la Patagonia centro-norte.

Es importante señalar que, aparte de la disponibilidad de nutrientes, la luz es un elemento clave en los procesos biogeoquímicos de los fiordos, especialmente, glaciales (Pantoja et al. 2010). Por lo tanto, los flujos y balances de nutrientes pueden verse significativamente alterados ante escenarios que incluyan un acelerado derretimiento glaciar y un incremento en el material en suspensión (SalcedoCastro et al. 2013). En este sentido, futuros estudios deberían incorporar estos procesos.
En conclusión, el modelo LOICZ demostró ser una herramienta útil para comprender la compleja dinámica de los nutrientes en áreas marinas proglaciales, el cual previamente solo había sido aplicado en sistemas estuarinos. Por otro lado, el uso de las simulaciones numéricas que permitan pronosticar futuros escenarios es cada vez más requerido para poder estimar los cambios inducidos por el calentamiento global (ver IPCC). E1 presente trabajo permitió determinar la influencia de la pluviosidad local en el flujo de nutrientes y a su vez estimar cómo las variaciones en el patrón de precipitaciones podrían afectar los procesos biogeoquímico en las áreas marinas proglaciales subantárticas.

\section{Agradecimientos}

Esta investigación fue financiada por el proyecto "Patterns in benthic communities off the Marinelli glacier (Darwin Ice Field, South Chile): Response to glacier retreat?" Fondo Nacional para la Ciencia y Tecnología (FONDECYT 11090208).

\section{LITERATURA CITADA}

Abreu PC, M Bergesch, LA Proença, C García \& C Odebrecht. 2010. Short- and long-term chlorophyll a variability in the shallow microtidal Patos Lagoon Estuary, Southern Brazil. Estuaries and Coasts 33: 554-569.

Andrade S. 1991. Geomorfología costera y antecedentes oceanográficos físicos de la Región de Magallanes, Chile $\left(48^{\circ}-56^{\circ} \mathrm{S}\right)$. Anales del Instituto de la Patagonia 20(1): $135-151$.

Barría K. 2016. Dinámica del flujo de nutrientes en un área marina proglacial (Seno Gallegos, Cordillera Darwin). Tesis de Biólogo Marino, Facultad de Ciencias, Universidad de Magallanes, Punta Arenas, 94 pp.

Borromei AM, MS Candel, LL Musotto, G Cusminsky, MA Martínez, CA Coviaga, JF Ponce \& A Coronato. 2018. Late Holocene wet/dry intervals from Fuegian steppe at Laguna Carmen, southern Argentina, based on a multiproxy record. Palaeogeography, Palaeoclimatology, Palaeoecology 499: 56-71.

Cabral A \& A Fonseca. 2019. Coupled effects of anthropogenic nutrient sources and meteo-oceanographic events in the trophic state of a subtropical estuarine system. Estuarine, Coastal and Shelf Science 225, 106228. <https://doi. org/10.1016/j.ecss.2019.05.010>

Calvete C \& M Sobarzo. 2011. Quantification of the surface brackish water layer and frontal zones in southern Chilean fjords between Boca del Guafo and Estero Elefantes. Continental Shelf Research 31(3-4): 162-171.

Castillo M. 2012. Circulación mezcla en el Fiordo Reloncaví, Chile. Tesis Doctoral en Oceanografía, Facultad de Ciencias y Oceanográficas, Universidad de Concepción, Concepción, $91 \mathrm{pp}$.

CENDHOC. 2010. Reporte de Datos de Cruceros Oceanográficos CIMAR. <http://www.shoa.cl/n_cendhoc> 
De Angelis H. 2014. Hypsometry and sensitivity of the mass balance to changes in equilibrium-line altitude: the case of the Southern Patagonia Icefield. Journal of Glaciology 60(219): 14-28.

Faranda FM, L Guglielmo \& P Povero. 1996. Straits of Magellan oceanographic cruise. Data Report, 281 pp. Ministry for Universities and Scientific and Technological Research, National Program for Antarctic Research, Genova.

Faviano M, P Puero, R Danovaro \& C Misic. 1999. Particulate organic matter composition in a semiclosed periantartic system: The Strait of Magellan. Scientia Marina 63(Suppl.1): 89-98.

Fernández RA, JB Anderson, JS Wellner \& B Hallet. 2011. Timescale dependence of glacial erosion rates: A case study of Marinelli Glacier, Cordillera Darwin, southern Patagonia. Journal of Geophysical Research: Earth Surface 116(1): 1-18.

Garreaud R, P Lopez, M Minvielle \& M Rojas. 2013. Largescale control on the Patagonian climate. Journal of Climate 26(1): 215-230. <https://doi: 10.1175/JCLI-D-12-00001.1>

Giordani G, PViaroli, DP Swaney, CN Murray, JM Zaldívar \& JI Marshall-Crossland. 2005. Nutrient fluxes in transitional zones of the Italian coast. LOICZ Reports \& Studies 28: 1-157. LOICZ, Texel.

González HG, MJ Calderón, L Castro, A Clement, L Cuevas, G Daneri, JL Iriarte, L Lizárraga, R Martínez, E Menschel, N Silva, C Carrasco, C Valenzuela, CA Vargas \& C Molinet. 2010. Primary production and its fate in the pelagic food web of the Reloncaví Fjord and plankton dynamics of the Interior Sea of Chiloé, Northern Patagonia, Chile. Marine Ecology Progress Series 402: 13-30.

Gordon DC Jr, PR Boudreau, KH Mann, JE Ong, WL Silvert, SV Smith, G Wattayakorn, F Wulff \& T Yanagi. 1996. LOICZ Biogeochemical Modelling Guidelines. LOICZ Reports \& Studies 5: 1-96. LOICZ, Texel.

Guzmán D. 2004. Caracterización hidrográfica, oceanográfica y balance de nitrógeno y fósforo del fiordo Aysén. Tesis de Magister en Oceanografía, Universidad de Valparaíso / Pontificia Universidad Católica de Valparaíso, Valparaíso, $76 \mathrm{pp}$.

Guzmán D \& N Silva. 2002. Caracterización física y química y masas de agua en los canales australes de Chile entre Boca del Guafo y Golfo elefantes (Crucero Cimar 4 Fiordos). Ciencia y Tecnología del Mar 25(2): 45-76.

Haro D, A Aguayo-Lobo \& J Acevedo. 2013. Características oceanográficas y biológicas de las comunidades del plancton y el necton del área marina costera protegida Francisco Coloane: una revisión. Anales del Instituto de la Patagonia 41(1): 77-90.

Hock R. 2003. Temperature index melt modelling in mountain areas. Journal of Hydrology 282(1-4): 104-115.

Iriarte JL, A Kusch, J Osses \& M Ruiz. 2001. Phytoplankton biomass in the sub-Antarctic area of the Straits of Magellan (53 S), Chile during spring-summer 1997/1998. Polar Biology 24(3): 154-162.

Kuylenstierna JL, GC Rosqvist \& P Holmlund. 1996. LateHolocene glacier variations in the Cordillera Darwin, Tierra del Fuego, Chile. The Holocene 6(3): 353-358.
López P, P Chevallier, V Favier, B Pouyaud, F Ordenes \& J Oerlemans. 2010. A regional view of fluctuations in glacier length in southern South America. Global and Planetary Change 71(1-2): 85-108.

Mansilla CA, RD McCulloch \& F Morello. 2018. The vulnerability of the Nothofagus forest-steppe ecotone to climate change: Palaeoecological evidence from Tierra del Fuego $\left(\sim 53^{\circ} \mathrm{S}\right)$. Palaeogeography, Palaeoclimatology, Palaeoecology 508: 59-70.

Montalvo J \& S Loza. 2006. Flujos de materiales conservativos y no conservativos en la Bahía de Jigüey (Archipiélago Sabana- Camagüey, Cuba) y el océano. Serie Oceanológica 2: 1-10.

Montero P, G Daneri, HE González, JL Iriarte, FJ Tapia, L Lizárraga, N Sánchez \& O Pizarro. 2011. Seasonal variability of primary production in a fjord ecosystem of the Chilean Patagonia: implications for the transfer of carbon within pelagic food webs. Continental Shelf Research 31: 202-215.

Pantoja S, JL Iriarte, MH Gutiérrez \& C Calvete. 2010. The southern Chile continental margin. In: Liu KK, L Atkinson, RA Quiñones \& L Talaue-McManus (eds). Carbon and nutrient fluxes in continental margins, pp- 265273. Springer, Berlin.

Pantoja S, JL Iriarte \& G Daneri. 2011. Oceanography of the Chilean Patagonia. Continental Shelf Research 31(34): $149-153$.

Pizarro G, JL Iriarte, V Montecino, JL Blanco \& L Guzmán. 2000. Distribución de la biomasa fitoplanctónica y productividad primaria máxima de fiordos y canales australes $\left(47^{\circ}-50^{\circ} \mathrm{S}\right)$. Ciencia y Tecnología del Mar 23: $25-48$.

Prado-Fiedler R. 2009. Winter and summer distribution of dissolved oxygen, $\mathrm{pH}$ and nutrients at the heads of fjords in Chilean Patagonia with possible phosphorus limitation. Revista de Biología Marina y Oceanografía 44(3): 783-789.

Prado-Fiedler R \& J Salcedo-Castro. 2008. Aporte fluvial y pluvial de nitrógeno y fósforo al fiordo Aysén y Canal Jacaf- Ventisquero-Puyuhuapi. Ciencia y Tecnología del Mar 31(2): 75-95.

Quiroga E, P Ortiz, D Gerdes, B Reid, S Villagran \& R Quiñones. 2012. Organic enrichment and structure of macrobenthic communities in the glacial Baker Fjord, Northern Patagonia, Chile. Journal of the Marine Biological Association of the United Kingdom 92(1): 73-83.

Quiroga E, P Ortiz, B Reid \& D Gerdes. 2013. Classification of the ecological quality of the Aysen and Baker Fjords (Patagonia, Chile) using biotic indices. Marine Pollution Bulletin 68(1-2): 117-126.

Ramesh R, Z Chen, V Cummins, J Day, C D'Elia, B Dennison, DL Forbes, B Glaeser, M Glaser, B Glavovic, H Kremer, M Lange, JN Larsen, M Le Tissier, A Newton, M Pelling, R Purvaja \& E Wolanski. 2015. Land-ocean interactions in the coastal zone: Past, present \& future. Anthropocene 12: 85-98.

Ramírez B. 2005. Distribución vertical de clorofila en los fiordos australes ubicados entre el estrecho de Magallanes y el cabo de Hornos (Chile). Ciencia y Tecnología del Mar 28(2): 43-61. 
Rivera A, T Benham, G Casassa, J Bamber \& J Dowdeswell. 2007. Ice elevation and areal changes of glaciers from the Northern Patagonia icefield, Chile. Global and Planetary Change 59(1-4): 126-137.

Salcedo-Castro J, D Bourgault, SJ Bentley \& B deYoung. 2013. Nonhydrostatic modeling of cohesive sediment transport associated with a subglacial buoyant jet in glacial fjords: a process-oriented approach. Ocean Modelling 63: 30-39.

Salcedo-Castro J, A Montiel, B Jara \& O Vásquez. 2015. Influence of a glacier melting cycle on the seasonal hydrographic conditions and sediment flux in a SubAntarctic Glacial Fjord. Estuaries and Coasts 38(1): 24-34.

Schlitzer R. 2016.Ocean Data View. <http://odv.awi.de>

Schneider W, I Pérez-Santos, L Ross, L Bravo, R Seguel \& F Hernández. 2014. On the hydrography of Puyuhuapi Channel, Chilean Patagonia. Progress in Oceanography 129: 8-18.

Sievers H \& R Prado. 1994. Contraste de las características oceanográficas del seno Aysén, Chile, entre invierno y verano (Lat. 45²0'S). Revista de Biología Marina 29(2): 167-209.

Sievers H, C Calvete \& N Silva. 2002. Distribución de características físicas, masas de agua y circulación general para algunos canales australes entre el golfo de Penas y el estrecho de Magallanes (Crucero CIMAR - Fiordo 2), Chile. Ciencia y Tecnología del Mar 25(2): 17-43.

Silva N. 2006. Oxígeno disuelto, $\mathrm{pH}$ y nutrientes en canales y fiordos australes. En: Silva N \& S Palma (eds). Avances en el conocimiento oceanográfico de las aguas interiores chilenas, Puerto Montt a cabo de Hornos, pp. 37-73. Comité Oceanográfico Nacional/Pontificia Universidad Católica de Valparaíso, Valparaíso.

Silva N \& S Palma. 2008. The CIMAR Program in the austral Chilean channels and fjords. In: Silva N \& S Palma (eds). Progress in the oceanographic knowledge of Chilean interior waters, from Puerto Montt to Cape Horn, pp. 11-15. Comité Oceanográfico Nacional/ Pontificia Universidad Católica de Valparaíso, Valparaíso.

Silva N \& CA Vargas. 2014. Hypoxia in Chilean Patagonian Fjords. Progress in Oceanography, 129: 62-74.

Silva N, C Calvete \& H Sievers. 1997. Características oceanográficas físicas y químicas de canales chilenos entre Puerto Montt y laguna San Rafael (crucero Cimar-Fiordo 1). Ciencia y Tecnología del Mar 20: 23-106.

Silva N, D Guzmán \& A Valdenegro. 2000. Aysén Sound, Chile. In: Smith SV, V Dupra, JI Marshall-Crossland \& CJ Crossland (eds). Estuarine systems of the South American region: carbon, nitrogen and phosphorus fluxes. LOICZ Reports \& Studies 15: 1-87. LOICZ, Texel.
Slaymaker O. 2011. Criteria to distinguish between periglacial, proglacial and paraglacial environments. Quaestiones Geographicae 30(1): 85-94.

Smith SV, V Dupra, JI Marshall Crossland \& CJ Crossland. 2000. Estuarine systems of the South American region: carbon, nitrogen and phosphorus fluxes. LOICZ Reports \& Studies 15: 1-87.

Swaney DP \& G Giordani. 2011. Proceedings of the LOICZ Workshop on biogeochemical budget methodology and applications, Providence, Rhode Island, November 9-10, 2007. LOICZ Research \& Studies 37: 1-195.

Swaney DP \& BH Hong. 2009a. The LOICZ budget toolbox documentation. Revision. <http://nest.su.se/mnode/ Toolbox/LOICZ_Toolbox_Documentation.doc $>$

Swaney DP \& BH Hong. 2009b. The LOICZ budget toolbox. Cornell University, New York. $<$ http://nest.su.se/mnode/ Toolbox/LOICZ_Toolbox.xls>

Torres R, M Frangópulos, M Hamamé, V Montecino, C Maureira, G Pizarro, B Reid, A Valle-Levinson \& J Blanco. 2011. Nitrate to silicate ratio variability and the composition of micro-phytoplankton blooms in the inner-fjords of Seno Ballena (Strait of Magellan, $54^{\circ} \mathrm{S}$ ). Continental Shelf Research 31: 244-253.

Valdenegro A \& N Silva. 2003. Caracterización oceanográfica física y química de la zona de canales y fiordos australes de Chile entre el Estrecho de Magallanes y Cabo de Hornos (CIMAR 3 FIORDOS). Ciencia y Tecnología del Mar 26(2): 19-60.

Valenzuela M \& S Avaria. 2009. Distribución del microfitoplancton marino entre el Seno Reloncaví y Boca del Guafo en invierno y primavera de 2005 (Crucero Cimar 11 Fiordos). Ciencia y Tecnología del Mar 32(2): 43-77.

Vásquez OJ. 2011. Evaluación espacial y temporal de la influencia de un glaciar sobre características oceanográficas en Seno Gallegos (Seno Almirantazgo, Chile). Tesis de Licenciado en Biología Marina, Universidad de Magallanes, Punta Arenas, 84 pp.

Vásquez OJ, S Pineda, E Quiroga, B Jara \& A Montiel. 2012. Relación entre clorofila-a y las variables oceanográficas en el área periglaciar del Seno Gallegos (Cordillera Darwin, Chile): Bajo condiciones invernales. Anales del Instituto de la Patagonia 40(1): 139-151.

Recibido el 13 de enero de 2020 y aceptado el 30 de julio de 2020

Editor asociado: Mauricio Landaeta D. 